\title{
Restricción de crecimiento aumenta el riesgo de displasia broncopulmonar, muerte y sepsis en gemelos de 30 o menos semanas de gestación
}

\author{
Growth restriction increases the risk of bronchopulmonary dysplasia, death, \\ and sepsis in twins of 30 weeks or less of gestation
}

Debora Sabatellia, Beatriz Milet ${ }^{\mathrm{b}}$, Patricia Mena ${ }^{\mathrm{c}, \mathrm{d}}$, Angélica Domínguez ${ }^{\mathrm{d}}$, Red Neonatal Neocosur

aHospital Juan A Fernández, Buenos Aires, Argentina

bClínica Alemana, Santiago, Chile

'Hospital Sótero del Río, Santiago, Chile

¿División de Pediatría, Escuela de Medicina, Pontificia Universidad Católica de Chile, Santiago, Chile.

Recibido el 18 de julio de 2018; aceptado el 11 de octubre de 2018

\section{Resumen}

Introducción: Múltiples factores influyen en el riesgo de morbimortalidad del prematuro con restricción del crecimiento intrauterino (RCIU). La comparación de gemelos con crecimiento intrauterino discordante permite evaluar su efecto, excluyendo factores maternos y manejo prenatal. Nuestro objetivo fue evaluar el efecto de la RCIU sobre la morbilidad aguda, crónica y mortalidad, en parejas de recién nacidos gemelares prematuros extremos. Pacientes y Método: Gemelos menores de $1500 \mathrm{~g}$ y 30 semanas de gestación, de la Red Neocosur. Se realizaron análisis separados de pares de gemelos concordantes, discordantes leves y severos, evaluando el efecto de la RCIU sobre morbi-mortalidad. Se realizó análisis multivariado para establecer magnitud del efecto. Resultados: 459 pares de gemelos, 227 concordantes, 110 discordantes leves y 122 severos. Entre los concordantes solo hubo diferencia en uso de oxígeno a las 36 semanas. En discordantes leves, el menor tuvo menos enfermedad de membrana hialina y requirió menos dosis de surfactante, pero tuvo un mayor riesgo de Displasia broncopulmonar (DBP) o muerte. En discordantes severos, el menor presentó mayor mortalidad, sepsis, utilización y permanencia en ventilación mecánica, pese a menor frecuencia de enfermedad de membrana hialina. En regresión múltiple, el riesgo combinado de DBP o muerte fue mayor en gemelo menor y discordante severo. Conclusión: En gemelos discordantes, la patología respiratoria aguda fue más frecuente en el gemelo mayor, aunque el riesgo de DBP o muerte fue mayor en el gemelo con RCIU.
Palabras clave:

Gemelos discordantes; restricción de crecimiento intrauterino; síndrome de dificultad respiratoria; enfermedad de membrana hialina; displasia broncopulmonar; prematurez; sepsis; mortalidad neonatal

Correspondencia:

Debora Sabatelli

debora.sabatelli@gmail.com 


\begin{abstract}
Introduction: Multiple factors influence the risk of morbidity and mortality of premature infants with intrauterine growth restriction (IUGR). The comparison of twins with different intrauterine growth allows evaluating the effect of the restriction, excluding maternal factors and prenatal management. Our objective was to assess the effect of IUGR on acute and chronic morbidity, and mortality of extreme preterm twins. Patients and Method: Twins weighing less than 1500 grams and gestation equal to or less than 30 weeks, of the Neocosur Network. Separate analyses were performed on concordant twin pairs, and on mild and severe discordant twins, evaluating the effect of IUGR on morbidity and mortality. A multivariate analysis was performed in order to establish the impact of this effect. Results: 459 twin pairs, 227 concordant twins, 110 of mild discordance, and 122 of severe discordance. Among the concordant ones, there was only a difference in oxygen uptake at 36 weeks. In those of mild discordance, the smaller twin presented a lower frequency of hyaline membrane disease and required fewer doses of surfactant, but had a higher risk of bronchopulmonary dysplasia (BPD) or death. In severe discordant twins, the smaller one presented higher mortality, sepsis, use and permanence in mechanical ventilation, despite the lower frequency of hyaline membrane disease. In multiple regression analysis, the combined risk of BPD or death was higher in the smaller twin and of severe discordance. Conclusion: In discordant twins, the acute respiratory pathology was more frequent in the larger one, although the risk of BPD or death was higher in the one with IUGR.
\end{abstract}

\section{Keywords:}

Discordant twins; intrauterine growth restriction; respiratory distress syndrome; hyaline membrane disease; bronchopulmonary dysplasia; prematurity; sepsis; neonatal mortality

\section{Introducción}

La restricción de crecimiento intrauterino (RCIU) es un factor de riesgo de morbimortalidad neonatal a toda edad gestacional, pero es crítica en el prematuro extremo. Generalmente, la morbilidad neonatal es de mayor frecuencia y gravedad en el niño con RCIU respecto al de buen crecimiento de igual edad gestacional, describiéndose una continua disminución en la morbilidad, a medida que aumenta el percentil de peso ${ }^{1}$.

El riesgo de morbilidad aguda, como enfermedad de membrana hialina; o crónica, como displasia broncopulmonar puede diferir en presencia de RCIU. En el caso de la enfermedad de membrana hialina se ha descrito discrepancias en el riesgo que confiere el RCIU ${ }^{2,3}$, aunque con diferencias entre mayores y menores de 32 semanas de edad gestacional ${ }^{4,5}$. Adicionalmente, otros factores, como el uso prenatal de corticoides, la morbilidad materna, el sexo, el tipo de parto y los eventos peri-parto también influyen en el riesgo.

El estudio de gemelos con crecimiento discordante permite comparar el efecto del crecimiento intrauterino sobre los resultados neonatales, en similares condiciones de morbilidad materna, edad gestacional y uso de corticoides. Nuestro objetivo fue evaluar el efecto de la RCIU sobre la morbilidad aguda, crónica y mortalidad en parejas de gemelos de edad gestacional menor o igual a 30 semanas y menos de $1.500 \mathrm{~g}$ de peso al nacer.

\section{Pacientes y Método}

La red neonatal Neocosur es una agrupación de Unidades de Neonatología de Argentina, Chile, Para- guay, Uruguay y Perú, que reúne información sobre la evolución de menores de $1500 \mathrm{~g}$ al nacer, desde el año $2001^{6}$. A partir de esta base de datos se realizó un estudio retrospectivo, descriptivo de casos y controles, de gemelares nacidos de embarazos múltiples entre los años 2001 y 2010, estableciendo pares de gemelos, siendo seleccionados finalmente aquellos con edad gestacional menor o igual de 30 semanas, minimizando que ambos tuvieran RCIU. En casos de embarazos múltiples de más de dos gemelos, para mantener la independencia entre duplas, se identificó uno al azar y, de cada dupla de cuatrillizos, dos al azar. Se definió discordancia según la fórmula:

$$
\begin{aligned}
& \text { (Peso gemelo mayor - Peso gemelo menor) / } \\
& \text { Peso gemelo mayor x } 100
\end{aligned}
$$

Los pares de gemelos con discordancia menor al $10 \%$ se consideraron concordantes, entre 10 a $20 \%$ se consideraron discordantes leves y sobre el $20 \%$ se catalogaron como discordantes severos. Se consignó antropometría, sexo, edad gestacional, mortalidad y morbilidades como: sepsis precoz, sepsis clínica (definida con elementos clínicos, de laboratorio y tratamiento antibiótico por más de 5 días, aunque hemocultivos sean negativos), número de éstas, ductus arterioso persistente diagnosticado después de las $48 \mathrm{~h}$, hemorragia intracraneana grados III y IV, enfermedad de membrana hialina (definida por radiografía alterada compatible con el diagnóstico y/o uso de surfactante), síndrome de dificultad respiratoria (definido como alteración de la frecuencia respiratoria, quejido, retracción subcostal durante el primer día de vida), uso de surfactante y número de dosis, enterocolitis necrotizante (consideran- 
do sólo aquellas grado II o más), perforación intestinal, uso y permanencia en ventilación mecánica y presión continua en vía aérea (CPAP), escape aéreo, días de oxigenoterapia, displasia broncopulmonar (DBP, definida como oxigenoterapia a las 36 semanas y alta con oxígeno suplementario.

Análisis Estadístico: Se realizaron análisis separados de pares de gemelos concordantes, discordantes leves y severos. Para cada par se identificó el gemelo de menor (caso) y mayor peso (control). Se usaron modelos mixtos para identificar las variables que se diferenciaban entre el gemelar mayor y menor, considerando la no independencia entre éstos. El identificador del par gemelar se utilizó como indicador de medida repetida y, usando un enlace logístico, se realizaron regresiones para ver el efecto del gemelar mayor/menor en las variables de interés. Adicionalmente, se repitió las regresiones ajustando por sexo. Para ver el efecto de las características del recién nacido (RN) sobre el resultado de DBP o muerte, se realizó un análisis multivariado donde se incluyeron todas las características del RN. La magnitud del efecto se cuantificó usando OR y su intervalo de $95 \%$ de confianza. Se fijó un nivel de significancia con un $\mathrm{p}<0,05$. Resultados expresados como media \pm DS. Se utilizó SPSS $17.0 \mathrm{y}$, para las regresiones logísticas, se usó la metodología "glmmML" del programa R versión 3.2.2. El estudio fue aprobado por el Comité de Ética del Servicio de Salud Sur Oriente en Santiago de Chile.

\section{Resultados}

Se identificaron 1.207 RN productos de embarazos múltiples, consistentes en 3 pares de cuatrillizos, 55 pares de trillizos y 515 pares de mellizos. Posteriormente se seleccionaron aquellos con 30 semanas o menos de gestación, resultando en 459 pares de gemelos. Ellos tuvieron una edad gestacional de 27,6 \pm 2,03 semanas, y 227 pares fueron concordantes, 110 pares fueron discordantes leves, y 122 pares, discordantes severos.

En el subgrupo concordante, la diferencia porcentual media de peso fue de $4,3 \%$ y de talla $0,9 \%$. El grupo de mayor peso, un porcentaje significativamente mayor fue de sexo femenino ( 55,50 vs $46,70 \%, \mathrm{p}=0,012)$. No hubo mayores diferencias en morbimortalidad, salvo una mayor frecuencia de administración de oxígeno a las 36 semanas de edad corregida en el gemelo menor $(22,5$ vs $13,9 \%$ en el gemelo mayor, $\mathrm{p}=0,009)$ (Tabla 1).

En el subgrupo de discordantes leves, el gemelar mayor tuvo un $14,3 \%$ más de peso y un 3,6\% más de

Tabla 1. Comparación entre gemelo mayor y menor entre pares concordantes

\begin{tabular}{|c|c|c|c|}
\hline & Gemelo Mayor & Gemelo Menor & Valor P \\
\hline $\begin{array}{l}\text { Características generales } \\
\text { Peso }(\mathrm{g}) \\
\text { Talla }(\mathrm{cm}) \\
\text { Mortalidad }(\%) \\
\text { Días de Hospitalización }\end{array}$ & $\begin{array}{c}1046,1 \pm 270,2 \\
35,7 \pm 3,3 \\
32,9 \\
50,1 \pm 45,7\end{array}$ & $\begin{array}{c}1000,8 \pm 260,3 \\
35,4 \pm 3,1 \\
31,4 \\
50,4 \pm 44,3\end{array}$ & $\begin{array}{c}<0,001 \\
0,006 \\
\text { NS } \\
\text { NS }\end{array}$ \\
\hline $\begin{array}{l}\text { Morbilidades respiratorias } \\
\text { SDR (\%) } \\
\text { Enfermedad de membrana hialina (\%) } \\
\text { Número de dosis de Surfactante } \\
\text { Uso de Ventilación mecánica (\%) } \\
\text { Permanencia en VM (días) } \\
\text { Uso de CPAP (\%) } \\
\text { Permanencia en CPAP (días) } \\
\text { DBP (\%) } \\
\text { DBP / Muerte (\%) } \\
\text { Uso de } \mathrm{O}_{2} \text { a las } 36 \text { semanas de EG (\%) } \\
\text { Alta con } \mathrm{O}_{2}(\%)\end{array}$ & $\begin{array}{c}86,4 \\
84,4 \\
1,29 \pm 1,02 \\
78,9 \\
7,8 \pm 14,2 \\
59,1 \\
8,6 \pm 8,7 \\
25,2 \\
55,5 \\
13,9 \\
5,9\end{array}$ & $\begin{array}{c}83,6 \\
83,9 \\
1,27 \pm 0,98 \\
76,5 \\
8,2 \pm 15,1 \\
60,0 \\
8,3 \pm 9,5 \\
29,1 \\
57,8 \\
22,5 \\
7,7\end{array}$ & $\begin{array}{l}\text { NS } \\
\text { NS } \\
0,347 \\
\text { NS } \\
\text { NS } \\
\text { NS } \\
\text { NS } \\
\text { NS } \\
\text { NS } \\
0,009 \\
\text { NS }\end{array}$ \\
\hline $\begin{array}{l}\text { Otras morbilidades } \\
\text { Sepsis precoz (\%) } \\
\text { Sepsis Clínica (\%) } \\
\text { Número de sepsis clínicas } \\
\text { DAP (\%) } \\
\text { ECN (\%) } \\
\text { HIC III/IV (\%) } \\
\text { ROP }(\%)\end{array}$ & $\begin{array}{c}3,3 \\
61,9 \\
0,95 \pm 1,1 \\
42,9 \\
11,3 \\
13,8 \\
23,5\end{array}$ & $\begin{array}{c}5,7 \\
68,3 \\
0,98 \pm 0,9 \\
49,0 \\
15,1 \\
13,2 \\
26,1\end{array}$ & $\begin{array}{l}\text { NS } \\
\text { NS } \\
\text { NS } \\
\text { NS } \\
\text { NS } \\
\text { NS } \\
\text { NS }\end{array}$ \\
\hline
\end{tabular}

SDR: Síndrome de dificultad respiratoria; VM: Ventilación mecánica; CPAP: Presión positiva continua en vía aerea; DBP: Displasia broncopulmonar; DAP: Ductus arterioso persistente; ECN: Enterocolitis necrotizante; HIC III/V: Hemorragia intracraneana grado III o IV; ROP: Retinopatía del prematuro; NS: no significativo 
Tabla 2. Comparación entre gemelo mayor y menor entre pares discordantes leves

\begin{tabular}{|c|c|c|c|}
\hline & Gemelo Mayor & Gemelo Menor & Valor $\mathrm{P}$ \\
\hline $\begin{array}{l}\text { Características generales } \\
\text { Peso }(\mathrm{g}) \\
\text { Talla }(\mathrm{cm}) \\
\text { Mortalidad }(\%) \\
\text { Días de Hospitalización }\end{array}$ & $\begin{array}{c}1118,3 \pm 268,9 \\
36,2 \pm 3,4 \\
22,2 \\
56,4 \pm 50,6\end{array}$ & $\begin{array}{c}958,5 \pm 232,0 \\
34,9 \pm 3,2 \\
27,3 \\
65,0 \pm 74,1\end{array}$ & $\begin{array}{l}0,0001 \\
0,0001 \\
\text { NS } \\
\text { NS }\end{array}$ \\
\hline $\begin{array}{l}\text { Morbilidades respiratorias } \\
\text { SDR (\%) } \\
\text { Enfermedad de membrana hialina (\%) } \\
\text { Número de dosis de Surfactante } \\
\text { Uso de Ventilación mecánica (\%) } \\
\text { Permanencia en VM (días) } \\
\text { Uso de CPAP (\%) } \\
\text { Permanencia en CPAP (días) } \\
\text { DBP (\%) } \\
\text { DBP / Muerte (\%) } \\
\text { Uso de } \mathrm{O}_{2} \text { a las } 36 \text { semanas de EG (\%) } \\
\text { Alta con } \mathrm{O}_{2}(\%)\end{array}$ & $\begin{array}{c}84,9 \\
89,7 \\
1,23 \pm 1,01 \\
71,0 \\
9,9 \pm 19,4 \\
70,1 \\
7,1 \pm 7,1 \\
29,1 \\
50,0 \\
21,4 \\
13,0\end{array}$ & $\begin{array}{c}77,4 \\
77,6 \\
0,92 \pm 0,98 \\
73,0 \\
8,6 \pm 14,2 \\
64,5 \\
8,7 \pm 11,9 \\
32,0 \\
56,7 \\
21,4 \\
7,4\end{array}$ & $\begin{array}{l}\text { NS } \\
0,01 \\
0,007 \\
\text { NS } \\
\text { NS } \\
\text { NS } \\
\text { NS } \\
\text { NS } \\
0,04 \\
\text { NS } \\
\text { NS }\end{array}$ \\
\hline $\begin{array}{l}\text { Otras morbilidades } \\
\text { Sepsis precoz (\%) } \\
\text { Sepsis Clínica (\%) } \\
\text { Número de sepsis clínicas } \\
\text { DAP (\%) } \\
\text { ECN (\%) } \\
\text { HIC III/IV (\%) } \\
\text { ROP }(\%)\end{array}$ & $\begin{array}{c}1,9 \\
70,5 \\
1,0 \pm 0,9 \\
45,7 \\
10,3 \\
8,1 \\
23,8\end{array}$ & $\begin{array}{c}3,4 \\
68,9 \\
1,2 \pm 1,2 \\
46,7 \\
15,0 \\
9,1 \\
32,5\end{array}$ & $\begin{array}{l}\text { NS } \\
\text { NS } \\
\text { NS } \\
\text { NS } \\
\text { NS } \\
\text { NS } \\
\text { NS }\end{array}$ \\
\hline
\end{tabular}

SDR: Síndrome de dificultad respiratoria; VM: Ventilación mecánica; CPAP: Presión positiva continua en vía aerea; DBP: Displasia broncopulmonar; DAP: Ductus arterioso persistente; ECN: Enterocolitis necrotizante; HIC III/IV: Hemorragia intracraneana grado III o IV; ROP: Retinopatía del prematuro; NS: no significativo

talla que el menor (ambos $\mathrm{p}=<0,0001)$, y un mayor porcentaje fue de sexo femenino (55,5 vs $41,8 \%$, $\mathrm{p}=0,02)$. El gemelo menor tuvo un menor frecuencia de enfermedad de membrana hialina (77,6 vs $89,7 \%$, $\mathrm{p}=0,01)$ y requirió un menor número de dosis de surfactante $(0,92 \pm 0,98$ vs $1,23 \pm 1,01, p=0,007)$, aunque tuvo un mayor riesgo combinado de muerte y DBP $(56,7$ vs $50 \%, \mathrm{p}=0,04)$ (Tabla 2 ).

En el subgrupo de discordantes severos, la diferencia porcentual media de peso fue de $33,2 \%$ y de talla 9,6\%. El gemelo menor presentó menor utilización y permanencia en CPAP (57,3 vs 70,1\%, p = 0,01, y 11,8 $\pm 11,8$ vs $8,7 \pm 11,3$ días, $p=0,008)$, pero mayor empleo y permanencia en ventilación mecánica ( 86,5 vs $76,6 \%$, $\mathrm{p}=0,027$, y $11,4 \pm 15,5$ vs $8,0 \pm 13,0$ días, $\mathrm{p}=0,023)$, pese a una menor frecuencia de enfermedad de membrana hialina ( 82,9 vs $90,6 \%, \mathrm{p}=0,035)$. Además tuvo mayor número de sepsis clínica $(1,18 \pm 1,22$ vs $0,82 \pm$ $0,83, \mathrm{p}=0,014)$ y mayor mortalidad $(39,3$ vs $26,2 \%$, $\mathrm{p}=0,01)$, sin diferencias en DBP y otras morbilidades (Tabla 3).

En gemelares concordantes encontramos 2,5 veces más riesgo que el gemelo menor requiera administración de oxígeno a las 36 semanas de edad corregida, persistiendo al ajustar por sexo. En gemelares discordantes leves, el gemelo menor presenta 3,3 veces menos riesgo de enfermedad de membrana hialina, incre- mentando a 3,6 al ajustar por sexo. Por el contrario, el RN mayor tuvo 0,08 veces menos riesgo combinado de DBP o muerte. En gemelares discordantes severos, se mantienen las asociaciones significativas (y el sentido de éstas) encontradas para los gemelares discordantes leves. A lo anterior se agrega que ser el gemelar mayor fue protector frente a Muerte o HIC severa $(\mathrm{OR}=0,26)$ y necesidad de VM $(\mathrm{OR}=0.33)$, y tuvo mayor riesgo de uso de CPAP $(\mathrm{OR}=2,71)$ (Tabla 4).

En la Tabla 5 se muestra el análisis multivariado del riesgo combinado de DBP o muerte de toda la muestra de gemelares, con mayor riesgo del gemelo menor, discordante severo, sexo masculino, menor edad gestacional y enfermedad de membrana hialina.

\section{Discusión}

El principal hallazgo de este estudio de gemelos prematuros menores de $1.500 \mathrm{~g}$, es que el menor crecimiento intrauterino se asocia a un mayor riesgo de muerte, DBP y sepsis. En gemelos concordantes, el menor tiene más riesgo de DBP, aún corrigiendo por edad gestacional, sexo o haber desarrollado enfermedad de membrana hialina. Tanto en discordantes leves como severos hubo mayor morbilidad aguda en el gemelo mayor, pero mayor morbilidad respiratoria crónica en 
Tabla 3. Comparación entre gemelo mayor y menor entre pares discordantes severos

\begin{tabular}{|c|c|c|c|}
\hline & Gemelo Mayor & Gemelo Menor & Valor P \\
\hline $\begin{array}{l}\text { Características generales } \\
\text { Peso }(\mathrm{g}) \\
\text { Talla }(\mathrm{cm}) \\
\text { Mortalidad }(\%) \\
\text { Días de Hospitalización }\end{array}$ & $\begin{array}{c}1143,8 \pm 206,7 \\
36,5 \pm 2,3 \\
26,2 \\
56,0 \pm 52,5\end{array}$ & $\begin{array}{c}764,4 \pm 161,5 \\
32,9 \pm 2,9 \\
39,3 \\
62,7 \pm 62,8\end{array}$ & $\begin{array}{c}0,0001 \\
0,006 \\
0 \\
\text { NS }\end{array}$ \\
\hline $\begin{array}{l}\text { Morbilidades respiratorias } \\
\text { SDR (\%) } \\
\text { Enfermedad de membrana hialina (\%) } \\
\text { Número de dosis de Surfactante } \\
\text { Uso de Ventilación mecánica (\%) } \\
\text { Permanencia en VM (días) } \\
\text { Uso de CPAP (\%) } \\
\text { Permanencia en CPAP (días) } \\
\text { DBP (\%) } \\
\text { DBP / Muerte (\%) } \\
\text { Uso de } \mathrm{O}_{2} \text { a las } 36 \text { semanas de EG (\%) } \\
\text { Alta con } \mathrm{O}_{2}(\%)\end{array}$ & $\begin{array}{c}91,5 \\
90,6 \\
1,40 \pm 1,04 \\
76,6 \\
8,0 \pm 13,0 \\
70,1 \\
8,70 \pm 11,3 \\
31,0 \\
53,5 \\
22,5 \\
8,5\end{array}$ & $\begin{array}{c}85,5 \\
82,9 \\
1,27 \pm 0,99 \\
86,5 \\
11,4 \pm 15,5 \\
57,3 \\
11,8 \pm 11,8 \\
34,5 \\
66,7 \\
27,5 \\
10,3\end{array}$ & $\begin{array}{c}\text { NS } \\
0,035 \\
\text { NS } \\
0,027 \\
0,023 \\
0,01 \\
0,008 \\
\text { NS } \\
0,06 \\
\text { NS } \\
\text { NS }\end{array}$ \\
\hline $\begin{array}{l}\text { Otras morbilidades } \\
\text { Sepsis precoz (\%) } \\
\text { Sepsis Clínica (\%) } \\
\text { Número de sepsis clínicas } \\
\text { DAP (\%) } \\
\text { ECN (\%) } \\
\text { HIC III/IV (\%) } \\
\text { ROP }(\%)\end{array}$ & $\begin{array}{c}1,8 \\
58,2 \\
0,82 \pm 0,83 \\
56,4 \\
8,5 \\
13,9 \\
33,8\end{array}$ & $\begin{array}{c}1,8 \\
68,7 \\
1,18 \pm 1,22 \\
48,7 \\
16,2 \\
11,1 \\
40,3\end{array}$ & $\begin{array}{l}\text { NS } \\
\text { NS } \\
0,014 \\
\text { NS } \\
\text { NS } \\
\text { NS } \\
\text { NS }\end{array}$ \\
\hline
\end{tabular}

SDR: Síndrome de dificultad respiratoria; VM: Ventilación mecánica; CPAP: Presión positiva continua en vía aerea; DBP: Displasia broncopulmonar; DAP: Ductus arterioso persistente; ECN: Enterocolitis necrotizante; HIC III/IV: Hemorragia intracraneana grado III o IV; ROP: Retinopatía del prematuro; NS: no significativo

Tabla 4. Regresiones logísticas para pares gemelares

\begin{tabular}{llc}
\hline Outcome & OR bruto (IC 95\%) & OR ajustado x sexo (IC 95\%) \\
\hline $\begin{array}{l}\text { Pares gemelares simétricos } \\
\text { Uso de } \mathrm{O}_{2} \text { a las } 36 \text { semanas de EG }\end{array}$ & $0,40(0,19-0,84)$ & $0,36(0,17-0,80)$ \\
Pares gemelares discordantes leves & & \\
$\quad$ Displasia/ muerte & $0,08(0,01-0,64)$ & $0,006(0,0002-0,19)$ \\
Enfermedad de membrana hialina & $3,33(1,26-8,85)$ & $3,68(1,34-10,15)$ \\
Pares gemelares discordantes severos & & \\
Mortalidad & $0,26(0,11-0,65)$ & $0,25(0,10-0,62)$ \\
Enfermedad de membrana hialina & $4,11(1,15-14,66)$ & $3,77(1,06-13,34)$ \\
Ventilación Mecánica & $0,33(0,12-0,89)$ & $0,29(0,10-0,84)$ \\
Ventilación de alta frecuencia & $0,10(0,02-0,42)$ & $0,10(0,02-0,42)$ \\
CPAP & $2,71(1,22-6,03)$ & $2,73(1,22-6,10)$ \\
Displasia o muerte & $0,29(0,12-0,72)$ & $0,27(0,11-0,68)$ \\
HIC severa o muerte & $0,32(0,14-0,76)$ & $0,31(0,13-0,73)$
\end{tabular}

EG: Edad gestacional; CPAP: Presión positiva continua en vía aérea ; HIC: hemorragia intra craneana; OR: odds ratio; IC 95\%: intervalo de $95 \%$ de confianza.

Tabla 5. Análisis multivariado para riesgo combinado de displasia broncopulmonar o muerte.

\begin{tabular}{|c|c|c|}
\hline Variable & OR (IC95\%) & Valor $p$ \\
\hline Gemelar mayor & $0,41(0,25-0,67)$ & $<0,001$ \\
\hline Par gemelar discordante leve & $1,91(0,70-5,21)$ & 0,203 \\
\hline Par gemelar discordante severo & $2,89(1,12-7,46)$ & 0,029 \\
\hline Sexo Masculino & $2,84(1,44-5,57)$ & 0,003 \\
\hline EG (semana adicional) & $0,23(0,16-0,33)$ & $<0,001$ \\
\hline Enfermedad de membrana hialina & $4,97(1,83-10,35)$ & 0,002 \\
\hline
\end{tabular}

EG: edad gestacional; OR: odds ratios; IC95\%: intervalo de 95\% de confianza. 
el gemelo menor, siendo más marcada en discordantes severos. En el análisis multivariado del total de gemelos, el menor tiene 2,44 veces más chances de DBP o muerte, ajustado por grado de asimetría del par gemelar, sexo, edad gestacional y enfermedad de membrana hialina. El gemelar menor que ha tenido enfermedad de membrana hialina tiene mucho mayor riesgo de DBP.

Los estudios de recién nacidos productos de embarazos múltiples pueden presentar diferentes resultados según cómo se efectúe la comparación. Gran parte de la literatura sobre gemelos discordantes analiza la decisión de interrupción del embarazo basado en el grado de discordancia y prematurez ${ }^{8}$. En este sentido, el análisis se presenta como productos de embarazos con o sin discordancia ${ }^{9,10}$ o con pequeños para edad gestacional $^{9}$ y sólo una minoría analiza el resultado de un gemelo con el otro ${ }^{11}$. La mortalidad fetal casi duplica la neonatal con discordancia mayor al $20 \%$ en gemelares menores de 32 semanas ${ }^{11}$.

En relación al riesgo neonatal, puede variar según definiciones de discordancia y evaluaciones de cigocidad, corionicidad, orden de nacimiento y crecimiento fetal ${ }^{8-10,12-17}$. En la comparación de crecimiento fetal se han utilizado variadas definiciones de discordancia, entre las cuales preferimos clasificarlos en leves y severos, para evaluar si las diferencias aumentaban con la mayor alteración del crecimiento.

En los embarazos gemelares, el riesgo de SDR se ha asociado con el sexo y el orden de nacimiento, siendo el varón más susceptible, y el nacido en segundo lugar con más riesgo para asfixia perinatal y falla respiratoria $^{9,18-20}$.

En nuestro estudio, la incidencia de DBP aumenta en los gemelos menores. Al controlar por sexo, los concordantes y discordantes leves tuvieron menos mujeres, pero la distribución fue similar en los discordantes severos. Interesantemente, se ha documentado que ante presencia de gemelos masculino y femenino, el primero ejerce un efecto masculinizante en el riesgo de problemas respiratorios. Así, los gemelos masculinos tienen mayor riesgo, el que es similar a los femeninos de embarazos masculino-femenino contrastando con los gemelares femenino-femenino ${ }^{21}$. También, habría un efecto del sexo de los gemelos sobre el peso de nacimiento. La presencia de un gemelo masculino se asocia a un mayor peso de nacimiento de gemelas femeninas que lo esperado sólo por género ${ }^{22}$.

Respecto a la presentación de SDR, los reportes

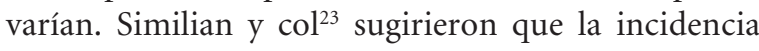
de SDR y taquipnea transitoria en prematuros discordantes es más alta que para los prematuros concordantes. La edad gestacional de los grupos comparados influye claramente en los resultados. En el mayor de 32 semanas, el ser pequeño para la EG (PEG) protege del riesgo de SDR pero no es evidente en menores ${ }^{4,5}$. En un estudio retrospectivo de 124 pares de gemelos encontraron que la necesidad de $\mathrm{O}_{2}$ por 4 horas en gemelos debajo de 28 semanas fue fuertemente asociado con ser el gemelo mayor ( $\mathrm{OR}=1,995 \%$ IC 1,03 $3,46)^{19}$. La causa por la que el gemelo mayor puede desarrollar más morbilidad pulmonar aguda podría estar dada por la posibilidad de que el gemelo menor se encuentre más maduro por el stress crónico de la RCIU, con producción endógena de corticoides, la cual es mayor al nacer en el discordante menor ${ }^{24}$. La RCIU se ha asociado con efectos adversos en la estructura del pulmón y la función resultando en una alteración persistente en la estructura del pulmón y una discapacidad de la función respiratoria en la vida postnatal (niños, adolescentes y adultos jóvenes) ${ }^{2}$. De esta manera se explica que el gemelo menor tenga mas patología respiratoria crónica.

Un análisis reciente de gemelos en la red canadiense de prematurez, según orden de nacimiento muestra una mayor frecuencia de PEG en el segundo gemelo (que se repite en otros estudios), pero mayor $\mathrm{SDR}^{14}$. La evolución según orden de nacimiento está modificada por la asociación entre segundo gemelar y menor crecimiento $^{25}$.

Pocos estudios realizan análisis por cigocidad, pero se ha usado la corionicidad y el sexo como equivalente, aunque ninguno de los dos es suficiente, describiéndose concordancia entre la asignación clínica y el estudio genético de sólo 62,7\% para gemelos monocigóticos y de un $88,9 \%$ para gemelos dicigóticos ${ }^{13}$. La cigocidad es una variable interviniente muy importante pero no es fácil de incluir si no se cuenta con estudios genéticos. Bhandari et al estudió cigocidad, pudiendo mostrar que existen factores genéticos asociados, atribuibles a la posibilidad de desarrollar $\mathrm{DBP}^{12}$.

Respecto a sepsis tardía los análisis de incidencia en relación al componente genético son controversiales, pero no parece que sea un factor claramente influyente. Gemelos dicigótos muestran un mayor riesgo de infección que los monocigotos ${ }^{26}$. En el caso de infección tardía se ha documentado que es poco probable que aspectos genéticos sean determinantes de este riesgo, dado la ausencia de diferencias entre productos únicos o múltiples y de la igual frecuencia entre gemelares del mismo y diferente sexo ${ }^{27}$. En cambio, el efecto del RCIU sobre el mayor riesgo de sepsis se ha documentado tanto en este como en otros estudios ${ }^{28}$. Así, es la RCIU la que aumenta el riesgo de infección. Esto, en parte se debería a que niños con RCIU presentan más requerimiento de catéteres venosos centrales, más tiempo en alcanzar alimentación enteral completa, más días y cursos de antibióticos que los niños de peso adecuado, mayor permanencia en ventilación mecánica y hospitalaria. Incluso controlando por todas estas 
variables, el RCIU sigue siendo un factor de riesgo de infección ${ }^{29}$. El compromiso inmunológico ha sido documentado en el recién nacido PEG desde hace muchos años ${ }^{30}$.

Las principales limitaciones de este estudio son no tener información de corionicidad y orden de nacimiento, ya que ambos factores modifican la morbilidad de los gemelos. Tampoco evaluamos el grado de compromiso de crecimiento del gemelo menor, respecto a las curvas de crecimiento intrauterino, influencia del sexo de los pares de gemelos, ni la sepsis documentada con hemocultivo positivo, aunque es posible que haya tenido comportamiento similar que la sepsis clínica.

\section{Conclusiones}

Existe mayor riesgo en gemelos con discordancia severa, donde el gemelo menor tiene menor riesgo de patología respiratoria aguda, pero más probabilidad de fallecer o presentar enfermedad pulmonar crónica, sepsis y hospitalización más prolongada.

\section{Responsabilidades éticas}

Protección de personas y animales: Los autores declaran que los procedimientos seguidos se conformaron a las normas éticas del comité de experimentación humana responsable y de acuerdo con la Asociación Médica Mundial y la Declaración de Helsinki.

Confidencialidad de los datos: Los autores declaran que han seguido los protocolos de su centro de trabajo sobre la publicación de datos de pacientes.

\begin{abstract}
Derecho a la Privacidad y Consentimiento Informado: Los autores declaran que la información ha sido obtenida de datos previos en forma anonimizada, por lo cual el Comité de Ética de Investigación en uso de sus facultades, ha eximido de la obtención de un consentimiento informado, lo cual consta en el acta respectiva.
\end{abstract}

\section{Conflicto de intereses}

Los autores declaran no tener conflicto de intereses.

\section{Referencias}

1. McIntire DD, Bloom SL, Casey BM, Leveno KJ. Birth Weight in relation to morbidity and mortality among newborn infants. N Eng J Med 1999;340:1234-8.

2. Manzanares S, Sánchez-Gila M, MorenoMartínez MD, Ramírez-Arredondo A, Pineda Perinatal outcomes in preterm growth-restricted twins: effects of gestational age and fetal condition. Twin Res Hum Genet. 2013;16(3):727-31.

3. Park CW, Park JS, Jun JK, Yoon BH. FGR in the setting of preterm sterile intra-uterine milieu is associated with a decrease in RDS. PediatrPulmonol. 2016;51(8):812-9.

4. Sharma P, McKay K, Rosenkrantz TS, et al. Comparisons of mortality and predischarge respiratory outcomes in small-for-gestational-age and appropriate-for-gestational-age premature infants. BMC Pediatr 2004; 4: 9.

5. Mena P, Smith J, Milet B, Toro C, Arredondo F, Llanos A. Incidencia y evolución de membrana hialina en menores de 35 semanas según crecimiento intrauterino. Rev Chil Pediatr 2011;82:395-401.

6. Grupo ColaborativoNeocosur. Verylow-birth-weight infant outcomes in 11 South American NICUs. JPerinatol. 2002;22(1):2-7.

7. Cerda J, Villarroel L. Evaluación de la concordancia inter-observador en investigación pediátrica: coeficiente de Kappa. Rev Chil Pediatr. 2008; 79 (1):548.

8. Patterson RM, Wood RC. What is twin birthweight discordance?Am J Perinat 1990;7:217-9.

9. Lopriore E, Sluimers C, Pasman SA, Middeldorp JM, Oepkes D, Walther FJ. Neonatal morbidity in growth-discordant monochorionic twins: comparison between the larger and the smaller twin. Twin Res Hum Genet. 2012;15(4):541-6.

10. Harper LM, Weis M, Odibo AO, Roehl KA, MaconesGA, Cahill, AG Significance of growth discordance in appropriately grown twins.Am J Obstet Gynecol. 2013;208(5):393.e1-5.

11. Wen SW, Fung KF, Huang L, et al. Fetal and Infant Health Group of the Canadian Perinatal Surveillance System. Fetal and neonatal mortality among twin gestations in a Canadian population: the effect of intrapair birthweight discordance. Am J Perinatol. 2005;22(5):279-86

12. Bhandari V, Bizzarro MJ, Shetty A, Zhong X, Page GP, Zhang H; Neonatal Genetics Study Group. Familial and genetic susceptibility to major neonatal morbidities in preterm twins. Pediatrics. 2006;117(6):1901-6.

13. Spiegler J, Härtel C, Schulz L, von Wurmb-Schwark N, Hoehn T, Kribs A; German Neonatal Network (GNN). Causes of delivery and outcomes of very preterm twins stratified to zygosity. Twin Res Hum Genet. 2012;15(4):532-6. .
14. Mei-Dan E, Shah J, Lee S, Shah PS, Murphy KE; Canadian NeonatalNetwork Investigators. The Effect of Birth Order on Neonatal Morbidity and Mortality in Very Preterm Twins.Am J Perinatol. 2017 Feb 23. doi: $10.1055 / \mathrm{s}-0037-1598255$

15. Steingass KJ, Taylor HG, Wilson-Costello D, Minich N, Hack M. Discordance in neonatal risk factors and early childhood outcomes of very low birth weight $(<1.5$ kg) twins. J Perinatol. 2013;33(5):388-93

16. Frezza S, Gallini F, Puopolo M, De Carolis MP, D’Andrea V, Guidone PI, Luciano R,Zuppa AA, Romagnoli C. Is growthdiscordance in twins a substantial risk factor in adverse neonatal outcomes? Twin Res Hum Genet. 2011;14(5):463-7.

17. Yinon $Y$, Mazkereth R, Rosentzweig N, Jarus-Hakak A, Schiff E, Simchen MJ. Growth restriction as a determinant of outcome in preterm discordant twins. Obstet Gynecol. 2005;105:80-4.

18. Steen EE, Källén K, Maršál K, Norman $\mathrm{M}$, Hellström-WestasL.Impact of sex on perinatal mortality and morbidity in twins. J Perinat Med. 2014;42(2):225-31

19. Canpolat FE, Yurdakok M, Korkmaz A, Yigit S, Tekinalp G. Birthweight discordance in twins and the risk of being heavier for respiratory distress syndrome. Twin Research and Human Genetics. 2006;9:659-63.

20. Webb RD, Shaw NJ. Respiratory distress in heavier versus lighter twins. Journal of Perinatal Medicine 2001;29:60-3.

21. Shinwell ES, Reichman B, Lerner-Geva 
L, Boyko V, Blickstein I; Israel Neonatal Network. "Masculinizing" effect on respiratory morbidity in girls from unlike-sex preterm twins: a possible transchorionic paracrine effect. Pediatrics. 2007;120:e447-53

22. Blumrosen E, Goldman RD, Blickstein I. Growth discordance and the effect of a male twin on birth weight of its female cotwin: a population-based study. J Perinat Med 2002; 30:510-3.

23. Smiljan Severinski N, Mamula O, Petrovic O. Neonatal outcome in discordant eutrophic twins: twin growth. Int J GynaecolObstet 2004;86:16-21.

24. Van Stralen G, vander Bos J, Lopriore E, Te Pas AB, Bloemenkamp. No shorttermbenefits of antenatal corticosteroid treatment in severely preterm growth restricted fetuses: A case-control study.
Early Human Develop. 2009;85:253-7.

25. Luo ZC, Ouyang F, Zhang J, Klebanoff M. Perinatal mortality in second- vs firstborn twins: a matter of birth size or birth order? Am J Obstet Gynecol. 2014;211(2):153.e1-8.

26. Härtel C, Schultz C, Herting E, Göpel W Genetic association studies in VLBW infants exemplifying susceptibility to sepsis-recent findings and implications for future research. Acta Paediatr. 2007;96(2):158-65.

27. Papiernik E, Zeitlin J, Delmas D, Blondel B, Künzel W, Cuttini M, MOSAIC Group. Differences in outcome between twins and singletons born very preterm: results from a population-based European cohort. Hum Reprod. 2010;25(4):103543.

28. Boghossian NS, Page GP, Bell EF, Stoll BJ,
Murray JC, Cotten CM; Eunice Kennedy Shriver National Institute of Child Health and Human Development Neonatal Research Network. Late-onset sepsis in very low birth weight infants from singleton and multiple-gestation births. J Pediatr. 2013;162(6):1120-4.

29. Tröger B, Göpel W, Faust K, Müller T, Jorch G, Felderhoff-Müser U; German Neonatal Network. Risk for late-onset blood-culture proven sepsis in verylow-birth weight infants born small for gestational age: a large multicenter study from the German Neonatal Network. Pediatr Infect Dis J. 2014;33(3):238-43.

30. Chandra RK. Serum thymic hormone activity and cell-mediated immunity in healthy neonates, preterm infants, and small-for-gestational age infants. Pediatrics. 1981;67(3):407-11. 